\title{
Biochemical and Immunological Characterizations of the Receptor Binding Domain of $C$. difficile Toxin B
}

\author{
Jui-Hsin Huang ${ }^{1,2}$, Chia-Wei Wu ${ }^{1}$, Shu-Pei Lien ${ }^{1}$, Kuang-Nan Hsiao ${ }^{1}$, Chih-Hsiang Leng ${ }^{1,3}$, lan Yu-Hsin Lin ${ }^{1}$ and Pele Chong ${ }^{1,3^{*}}$ \\ ${ }^{1}$ Vaccine R\&D Center, National Institute of Infectious Diseases and Vaccinology, National Health Research Institutes, Zhunan Town, Miaoli County, Taiwan \\ ${ }^{2}$ Graduate Institute of Life Science, National Defense Medical Center, Taipei, Taiwan \\ ${ }^{3}$ Graduate Institute of Immunology, China Medical University, Taichung, Taiwan
}

"Corresponding author: Pele Chong, Vaccine R\&D Center, National Institute of Infectious Diseases and Vaccinology, National Health Research Institutes, Zhunan Town, Miaoli County, Taiwan, Tel: 350-886-37-246-166; Email: pelechong@nhri.org.tw

Received date: 06 January 2015; Accepted date: 15 March 2015; Published date: 20 March 2015

Copyright: (C) 2015 Huang J, et al. This is an open-access article distributed under the terms of the Creative Commons Attribution License, which permits unrestricted use, distribution, and reproduction in any medium, provided the original author and source are credited.

\begin{abstract}
Clostridium difficile $(\mathrm{Cd})$ is an emerging nosocomial pathogen responsible for antibiotic-associated pseudomembranous colitis and diarrhea in hospital acquired infections. Clostridial toxins $A(T c d A)$ and $B($ TcdB) which specifically bind to unknown glycoprotein(s) on the surface of epithelial cells disrupt the intestinal barrier and ultimately lead to acute inflammation and diarrhea. There is still debate as to whether the receptor binding domains (RBD) of toxins can individually elicit protection in the hamster challenge model. In this study, a TcdB RBD which was derived from $C$. difficile strain VPI10463 with $>95 \%$ amino acid sequence identity to hyper-virulent strain BI/ NAP1/027 was designed and expressed in Escherichia coli. Recombinant RBD (rRBD) was purified, characterized biologically and immunologically and found to have the following properties: (a) capable of binding to the cell surface of both Vero and Caco-2 cells and entering into the cytosol; (b) devoid of hemagglutinin activity (HA); (c) the ability to up-regulate cell surface markers expressions and cytokines secretions from dendritic cells; (d) eliciting anti-TcdB neutralizing antibody responses that could weakly cross-neutralize TcdA in the absence of adjuvant; (e) and inducing weak protection against a lethal dose of $\mathrm{Cd}$ spores in the hamster challenge model. Therefore, rRBD shows potential as an immunogen to be included in the development of vaccines against Clostridium difficileassociated diseases.
\end{abstract}

Keywords: $C$. difficile infection; Antibiotic-associated pseudomembranous colitis; $C$. difficile toxins; Receptor binding domain; Toll-like receptor agonist; Vaccine

\section{Introduction}

In the past two decades, because of an increase in multi-drug resistant strains, Clostridium difficile-associated diseases (CDAD), such as diarrhea, pseudomembranous colitis, and toxic megacolon have become serious emerging infectious diseases worldwide [1-3]. A hyper-virulent and antibiotic-resistant epidemic strain NAP1/027 recently found in developed countries poses a major challenge for CDAD prevention since strain VP110463 had been used as the standard in most current vaccine development [4,5]. More importantly, $C$. difficile relapse was found to be around $15-35 \%$ within a few weeks after the standard therapy using either vancomycin and/or metronidazole [6]. The pathogenicity of $C$. difficile infections (CDI) is largely correlated to clostridial toxins A and B (TcdA and TcdB) which are secreted in the gastrointestinal environment of infected hosts and disrupt the epithelial cell barriers in the small intestine [7]. The mechanism underlying TcdA and TcdB toxicity involves three steps: (a) binding to unidentified receptor protein(s) on the surface of intestinal epithelium and internalization through its C-terminal receptor binding domain, (b) auto-cleavage and translocation of the $\mathrm{N}$-terminal glucosyltransferase domain from the endosomal membrane to the cytosol; and (c) the N-terminal enzymatic region that inactivates the Rho GTPase family by glycosylation [8].
TcdA-specific antibodies in patient sera were found to positively correlate with the prevention of CDAD recurrence [9-12], so TcdAspecific monoclonal antibodies are currently being tested in clinical trials [13-15]. In addition, different $C$. difficile vaccine strategies are being evaluated; the most advanced being vaccination with formalininactivated toxins $[9,16-19]$. Immunization with the receptor binding domain (RBD) of TcdA as antigens formulated with different adjuvant has been shown to elicit toxin-neutralizing antibody responses and protect mice from toxin challenges [20-26]. TcdB RBD has been less studied. A recent publication [27] has indicated that recombinant fragment of TcdB RBD in the presence of adjuvant could elicit neutralizing antibody responses against $\mathrm{TcdB}$ but not $\mathrm{Tcd} A$, only vaccine candidates containing both RBDs from TcdA and TcdB would confer protection in an in vivo hamster challenge model. Dingle et al. [28] also showed recombinant TcdB RBD to have poor biological functions such as not being able to agglutinate red blood cells, or to compete and directly block the cytotoxicity of TcdB and TcdA.

TcdB RBD is predicted to have a molecular size of approximately 63 $\mathrm{kDa}$ and is composed of 22-24 homologous repetitive peptides which may contain 4 potential lectin-like receptor-binding sites $[8,22,28,29]$. The specific roles and functions of these 4 putative binding regions are unclear, but they correlate with the lectin-like binding to the synthetic oligosaccharide, Gala1-3Galß1-4GlcNAc [28-30]. In this study, a consensus sequence of TcdB RBD was identified from different $C$. difficile strains deposited in the NCBI protein database. A consensus recombinant TcdB RBD (rRBD) was designed and expressed in Escherichia coli; the purified rRBD was characterized biologically and 
immunologically to test its merits as a potential vaccine candidate against CDAD.

\section{Materials and Methods}

\section{Ethics statement}

All experiments were conducted in accordance with the guidelines of the Laboratory Animal Center of National Health Research Institutes (NHRI). Animal use protocols have been reviewed and approved by the Institutional Animal Care and Use Committee of National Health Research Institutes (Approved protocol No. NHRIIACUC-100053-A).

\section{Construction of TcdB RBD}

The identification and design of the consensus sequence for TcdB RBD were performed according to a methodology described previously [31]. Briefly, TcdB RBD sequences from different Clostridium difficile strains deposited in the NCBI database were aligned for sequence analysis using the alignment tools from Vector NTI Advance 11.5 (Life technologies, Carlsbad, CA). This consensus sequence was analyzed by online software (http://www.ebi.ac.uk/ Tools/pfa/radar/) for the detection of repetitive protein sequences and potential ligand binding sites. The nucleotide sequence of tcdB rRBD coding for the C-terminal 537 amino acids of TcdB was optimized for E. coli codon usage (Figure 1), chemically synthesized (GeneArt; Life technologies) for cloning and expressed in E. coli. Gene encoding for TcdB RBD was inserted into a pET-22b vector (Novagen, Darmstadt, Germany) containing a polyhistidine tag coding sequence at the 3 '-end before the NdeI and XhoI restriction sites. The resulting pET-22b_tcdB RBD construct was transformed into E. coli JM109 (DE3) (Promega, Madison, WI) for TcdB RBD (rRBD) expression.

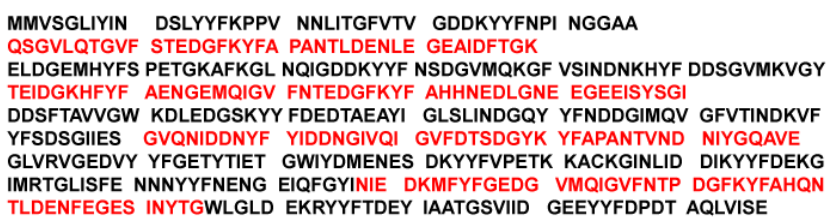

Figure 1: Amino acid sequence of the C-terminal repetitive and putative receptor binding domain from $C$. difficile toxin $\mathrm{B}$ (TcdB $\mathrm{RBD})$. (A) The amino acid sequence (537 residues) of TcdB RBD was identified using the online software (http://www.ebi.ac.uk / Tools/pfa/radar/). The localization of the 4 long-repeat (LR) regions of oligossacharide-binding sites are highlighted in red color.

\section{Production of rRBD}

rRBD was expressed in E. coli JM109 (DE3) (Promega) grown in LB medium at 20 oC for 16 hours following induction with $1 \mathrm{mM}$ isopropyl- $\beta$-D- thiogalacto-pyranoside (IPTG). The purification process for rRBD is briefly described below. Cells from 2 liters of culture medium were harvested by centrifugation and stored at $-20^{\circ} \mathrm{C}$ before re-suspension in lysis buffer $(50 \mathrm{mM}$ sodium phosphate buffer, pH 7.2 containing $250 \mathrm{mM} \mathrm{NaCl}$ and $5 \mathrm{mM}$ imidazole). The cells were physically disrupted using a French Press (Constant System, Daventry, UK) at $27 \mathrm{Kpsi}$ and the supernatant was collected by filtration through a $0.22 \mu \mathrm{m}$ filter. The crude extract was directly applied onto a nickel affinity chromatography column (GE Healthcare, Uppsala, Sweden) for purification of rRBD at $4^{\circ} \mathrm{C}$. After sequential washes with low concentrations of imidazole buffer, rRBD was eluted using a lysis buffer containing $500 \mathrm{mM}$ imidazole. The eluent was then dialyzed in a $30 \mathrm{kDa}$ cut-off dialysis bag against phosphate buffered saline (PBS), pH 7.2 containing $10 \%$ glycerol. To remove bacterial endotoxin, the rRBD solution was passed through an E membrane (Pall Corporation, Ann Arbor, MI). All purification steps were analyzed by $8 \%$ SDSPAGE. The residual endotoxin was determined using the Limulus amoebocyte lysate (LAL) assay (Associates of Cape Cod, Inc., Cape Cod, MA).

\section{Circular dichroism analysis}

The purified rRBD was diluted in $10 \mathrm{mM}$ phosphate buffer ( $\mathrm{pH} 7.0$ ) at a concentration of $50 \mu \mathrm{g} / \mathrm{mL}$. Spectra were obtained using a J-185 spectropolarimeter (Jasco, Easton, MD) with temperature control, and data were acquired in continuous scanning mode using a path length of $0.5 \mathrm{~mm}$, at a $0.2 \mathrm{~nm}$ interval and an accumulation time of 10-15 s/ $\mathrm{min}$. The far UV scan range was set between 190-250 nm with a scan speed of $50 \mathrm{~nm} / \mathrm{min}$. All data were processed using Jascot software, and a background spectrum obtained from PBS buffer was subtracted from the acquired sample spectrum. For the thermal stability test, the temperature gradient was set between 30 and $95^{\circ} \mathrm{C}$. CD spectra were recorded at an interval of $5^{\circ} \mathrm{C}$. The percent of the protein structure that was helical was calculated from mean residue ellipticity $([\theta])$ at $208 \mathrm{~nm}$ according to Freenfield and Fasman [32].

\section{Immuno-fluorescence staining and confocal microscopy for cell-binding and entry analysis}

Vero cells were seeded in 75T flasks with VP-SFM containing $4 \mathrm{mM}$ glutamine at $37^{\circ} \mathrm{C}, 5 \% \mathrm{CO}_{2}$, and allowed to grow to $80 \%$ confluency. Cells were isolated and suspended with VP-SFM culture media at $2 \times$ $10^{5}$ cells $/ \mathrm{mL}$ inside the flow tube. Resuspended cells were mixed with final $80 \mu \mathrm{g} / \mathrm{mL}$ of $\mathrm{rRBD}$ at $4^{\circ} \mathrm{C}$ for 5,15 , and $30 \mathrm{~min}$. After washing three times with cold PBS buffer, cells were sequentially fixed and then permeabilized with buffers specific for flow analysis (eBioscience, San Diego, CA). Anti-Fc receptor antibody (BD science) was added for 10 min before stepwise incubation with the specific anti-TcdB antibody (GeneTex) and a secondary antibody conjugated with fluorescein isothiocynate (FITC) (Sigma-Aldrich) on ice for $30 \mathrm{~min}$. At every step, cells were washed with cold PBS three times. After the last PBS washing step, cells were further washed with double distilled $\mathrm{H}_{2} \mathrm{O}$ to remove salt. Cells were simultaneously mounted onto glass slides, and nuclei stained with DAPI (Invitrogen, Carlsbad, CA.) and preparations subjected to $-20^{\circ} \mathrm{C}$ before confocal microscopy analysis (Leica TCS SP5 II; Leitz, Heidelberg, Germany).

\section{Fluorescence-activated cell sorting flow cytometry (FASC)}

Vero cells were seeded in $75 \mathrm{~T}$ flasks containing VP-SFM/4mM glutamine and allowed to grow to $80 \%$ confluentcy at $37^{\circ} \mathrm{C}$. An aliquot of resuspended cells $\left(5 \times 10^{5}\right.$ cells) were mixed with $50 \mu \mathrm{g} / \mathrm{mL}$ of $\mathrm{rRBD}$ at $4^{\circ} \mathrm{C}$ for 5 minutes. After washing with cold PBST, $1 \mu \mathrm{g}$ of either anti-TcdA antibody PCG-4 or anti-his tag antibody (AbD Serotec, Oxfordshire, UK) was added to the cells and the mixture was incubated on ice for $30 \mathrm{~min}$. After washing twice with cold PBS, an FITC-conjugated secondary antibody (Sigma-Aldrich, St. Louis, MO.) was added and mixed for $30 \mathrm{~min}$ for surface staining. Before flow 
cytometry analysis, propidium iodide (Sigma-Aldrich) was added to assess cell viability.

\section{Hemagglutinin activity assay}

Hemagglutinin (HA) activity assay was performed as described by Wren et al. [33]. In brief, 1 nmole of rRBD or 50 pmole of TcdA RBD (positive control) in $25 \mu \mathrm{L}$ used as the starting samples, were serially diluted two-fold with PBS buffer in 96-well round-bottom plates. A 25 $\mu \mathrm{L}$ of a $2 \%$ of rabbit erythrocyte (pre-washed with $\mathrm{PBS}$ ) suspension was added into the wells. The mixtures were incubated at $4^{\circ} \mathrm{C}$ overnight. HA activity was calculated by visual scoring.

\section{Mouse immunogenicity study}

$\mathrm{BALB} / \mathrm{c}$ mice were purchased from the National Animal Center in Taiwan and held in the Animal Center of the NHRI. The general immunization protocol is briefly described below. Three groups of mice (6 $\mathrm{BALB} / \mathrm{c}$ mice per group) were vaccinated with three intramuscular injections of 0,3 or $10 \mu \mathrm{g}$, respectively, of rRBD every two weeks. Before each immunization, mice were bled to collect sera that were stored at $-20^{\circ} \mathrm{C}$ before being used for anti-RBD antibody titer determination using RBD-specific ELISA and anti-TcdB neutralization assay as described below.

\section{Antigen-specific ELISA}

ELISA plate wells were coated with $100 \mathrm{ng}$ of rRBD overnight and blocked with $5 \%$ nonfat dry milk (w/v) in PBS. Mouse antisera serially diluted 2-fold with PBS containing 1\% BSA (Calbiochem, Darmstadt, Germany) were added to the wells followed by incubation at room temperature (RT) for 2 hours. After washing with $3 \times$ PBST, either anti-IgG isotypes (Invitrogen, Carlsbad, CA.) or HRP-conjugated IgG (KPL, Gaithersburg, MD) specific antibodies diluted in PBS containing $1 \%$ BSA were added to the wells and incubated at RT for 1 hour. After washing with $3 \times$ PBST, the plates were treated with TMB peroxidase substrate (KPL) at room temperature in the dark for 20 min. To determine anti-RBD titers, $\mathrm{OD}_{450} \mathrm{~nm}$ absorbance was measured using a spectrophotometer. End-point serum titers were defined as maximum dilution representing 0.2 cut-off value of $\mathrm{OD}_{450}$ $\mathrm{nm}$ measurement. This cut-off value was determined as the mean plus two standard deviations of the mean optical density value from six pre-immunized mouse sera diluted at a 1:20. The maximum dilution of each serum sample was finally represented as geometric mean titer (GMT).

\section{Anti-TcdB neutralization assay}

The anti-TcdB neutralization assay was performed according to the protocol previously described by Huang et al. [31]. Briefly, Vero cells $\left(2 \times 10^{4}\right.$ per well $)$ were seeded into 96 -well plates containing VP-SFM culture medium and $4 \mathrm{mM}$ glutamine at $37^{\circ} \mathrm{C}$, and allowed to grow to confluentcy Sera either from mice or hamsters immunized with rRBD were serially diluted two-fold with fresh VP-SFM and mixed with an equal volume of $40 \mathrm{pg} / \mathrm{mL}$ toxin B (The Native Antigen Company Ltd, Oxfordshire, UK) and incubated at room temperature for 1 hour. The mixture was added to the 96-well plates containing Vero cells and incubated at $37^{\circ} \mathrm{C}$ for 24 hours. Anti-TcdB neutralization titers were calculated as the highest serum dilution which could prevent $50 \%$ of cell from rounding due to $\mathrm{TcdB}$ cytotoxicity. Cellular toxicity was recorded using a microscope equipped with a camera.

\section{Surface markers and cytokines analyses for DC maturation}

Analysis of DC maturation was performed in vitro as previously described [34]. C57BL/6 mice were purchased from National Animal Center in Taiwan and held in the Animal Center of the NHRI. In brief, bone marrow-derived DCs (BMDCs) were collected from the tibiae of 6 to 8-week old C57BL/6 females. Bone marrow cells were isolated by vigorously washing with LCM (RPMI 1640 containing 1\% antibiotics with penicillin and streptomycin, $10 \%$ heat-inactivated FBS, $50 \mu \mathrm{M} \beta$ mercaptoethanol, and $50 \mathrm{mM}$ HEPES) and treated with lysis buffer to remove erythrocytes. BMDC were re-suspended at $2 \times 10^{6}$ cells per $\mathrm{mL}$ in LCM and treated with $20 \mathrm{ng} / \mathrm{mL}$ of recombinant granulocyte macrophage colony stimulating factor (MoGM-CSF) (Peprotech, Rocky Hill, NJ) on days 0 and 3. An aliquot of suspended BMDCs equivalent to $2 \times 10^{6}$ cells $/ \mathrm{mL}$ was seeded into 24 -well plates on day 6 . Varying concentrations of rRBD with or without $10 \mathrm{ng}$ of polymyxin $\mathrm{B}$ were added to the wells. LPS (1000 EU, Sigma-Aldrich) served as control. After 16 to $18 \mathrm{hr}$ incubation at $37^{\circ} \mathrm{C}$, BMDCs were analyzed by flow cytometry (FACSCalibur, BD Biosciences, Franklin Lakes, NJ) to evaluate the up-regulation of cell surface markers. In order to exclude immature DCs, representing $50 \%$ of the total cell population, the $\mathrm{CD} 11 \mathrm{c}^{+}$cell population was gated for surface marker staining with specific monoclonal antibodies to CD40, CD80, CD86, and MHC-II. In addition, cell culture supernatants were collected for cytokine expression. Cytokines such as IL-6, IL-12p40 and TNF- $\alpha$ were determined using specific cytokine kits purchased from eBioscience (San Diego, CA ).

\section{Preparation of $C$. difficile spores and hamster challenge model}

The protocol for preparation of $C$. difficile spores was modified from Lyras et al., [35]. Briefly, $C$. difficile strains VPI10463 were streaked on 10 anaerobic blood agar plates and grown anaerobically at $37^{\circ} \mathrm{C}$ to induce sporulation at around 5 to 6 days. The cells were harvested with disposable loops and washed in $10 \mathrm{~mL}$ PBS, and heatshocked at $56^{\circ} \mathrm{C}$ for $30 \mathrm{~min}$ to kill surviving vegetative cells. The spores were collected by low-speed centrifugation and resuspended in DMEM, aliquoted and frozen at $-80^{\circ} \mathrm{C}$. The frozen spores were then quantified before use by plating ten-fold serial dilutions of the spores onto Taurocholatefructose-agar (TFA) plates which were prepared with agar plus taurocholate-cefoxitin- cycloserinefructose-agar (TCCFA) without cycloserine and cefoxitin. The hamster challenge model was performed as follows. Six hamsters per group (6 weeks old and weighing 100-130 g) purchased from National Animal Center in Taiwan and held in the Animal Center of the NHRI were given clindamycin orogastrically $(30 \mathrm{mg} / \mathrm{kg})$ to render them susceptible to $C$. difficile infection (day 0 ). On day-5 post clindamycin treatment hamsters in each group were gastrically inoculated with 100 c.f.u. of $C$. difficile, and monitored twice daily for 5 days and daily thereafter. Animal bedding was changed and faecal pellets were collected daily. Specimens were inoculated onto selective TCCFA plates and incubated anaerobically at $37^{\circ} \mathrm{C}$ to determine if they were colonized with $C$. difficile. Faecal pellets were collected daily for 12 days, then weekly until the study terminated (at least 14 days). Each hamster group was assessed for $C$. difficile colonization and survival rate.

\section{Statistical analysis}

Data were expressed using Prism 5 version 5.01 (GraphPad Software, Inc.). Antibody titer was displayed as means \pm SEM from the experiments. Statistical difference was analyzed using two-tailed 
students' t-test by comparison of the means obtained in each treatment with the control group. The p-value $<0.05$ is considered to be significant.

\section{Results}

\section{Design of TcdB RBD}

According to previous studies [22,27-28,30,35], the receptor binding domain of TcdB is located between the C-terminal residues 1834 to 2366 and has a molecular weight of approximately $63 \mathrm{kDa}$. Sequences of $\mathrm{TcdB} \mathrm{RBD}$ based on different $C$. difficile isolates deposited in the NCBI protein database including VPI10463 and ATCC9689 which are the reference strains for $C$ difficile toxin studies and BI/NAP1/027 hyper-virulent strains were aligned and examined. The results indicated that the TCdB RBD amino acid sequences are conserved between these strains with $90-97 \%$ identity that is consistent with those previously reported by Stabler et al. [36]. The selected amino acid sequence of TcdB RBD is identical to that of $C$. difficile strains VPI10463 and composed of 537 residues (Figure 1). Based on the crystal structure reported by $\mathrm{Ho}$ et al. [29], this sequence comprises four potential oligosaccharide receptor-binding sites with core sequences QxGVFxTEDGFKYFA xxN as shown in Figure 1.

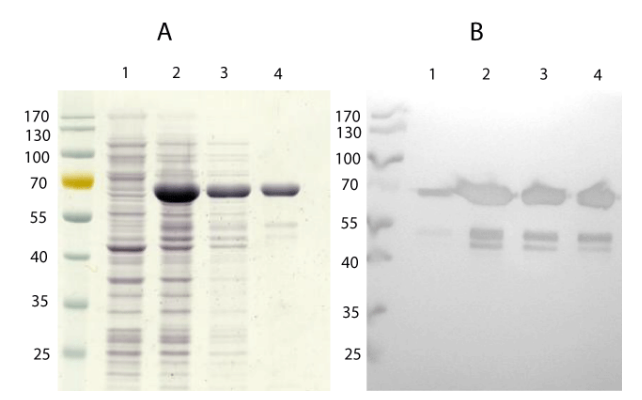

Figure 2: The expression and the purity of rRBD were confirmed by SDS-PAGE analysis (A) and Western blotting using toxin Bspecific monoclonal antibody. (B). Purity of rRBD is shown in panel. A: lanes 1 to 4 correspond to cell lysate before IPTG induction, cell lysate after IPTG induction, supernatant of IPTGinduced cell lyseis, and eluent of $500 \mathrm{mM}$ imidazole, respectively. The first lane contains molecular markers.

\section{Production of recombinant TcdB RBD (rRBD)}

The coding sequence of rRBD was designed using codon usage optimization, chemically synthesized, inserted into pET-22b vector and successfully expressed in E. coli as shown in Figure 2. After singlestep purification using $\mathrm{Ni}$-affinity chromatography, highly purified rRBD ( $>95 \%$ purity) was obtained and its purity confirmed by SDSPAGE (Figure 2A), and the Western blot analysis using anti-TcdB specific monoclonal antibody (Figure $2 \mathrm{~B}$ ). The degradation products are likely the result of proteolytic digestion during the purification process. In any event, at least $20 \mathrm{mg}$ of highly enriched rRBD (Figure 2B lane 3) could be easily obtained from 0.5 liter of bacterial culture. Most of the E. coli endotoxin (LPS) was successfully removed by passing the rRBD preparation through an E membrane. Residual LPS in the purified rRBD was found to be below $0.01 \mathrm{EU}$ per $\mu \mathrm{g}$ of protein based on the Limulus assay. We found that the best condition for preserving rRBD integrity was to store the protein at $3 \mathrm{mg} / \mathrm{mL}$ in PBS containing $10 \%(\mathrm{v} / \mathrm{v})$ of glycerol at $-80^{\circ} \mathrm{C}$.

\section{Secondary structure analysis by circular dichroism (CD)}

The secondary protein structure of $\mathrm{rRBD}$ was investigated and analyzed using circular dichroism. Figure $3 \mathrm{~A}$ shows the CD spectra of rRBD at room temperature. According to the method developed by Freenfield and Fasman [32], the major secondary protein structure of $\mathrm{rRBD}$ is estimated to contain $47 \%$ of $\beta$-sheet structure; this is consistent with other reports that the fragments of TcdA RBD form stable $\beta$-solenoid secondary structures [29,31]. The CD secondary structure analysis supports that rRBD was correctly folded to form a stable $\beta$-solenoid structure that melts at $49^{\circ} \mathrm{C}$ (Figure $3 \mathrm{~A}$ ).

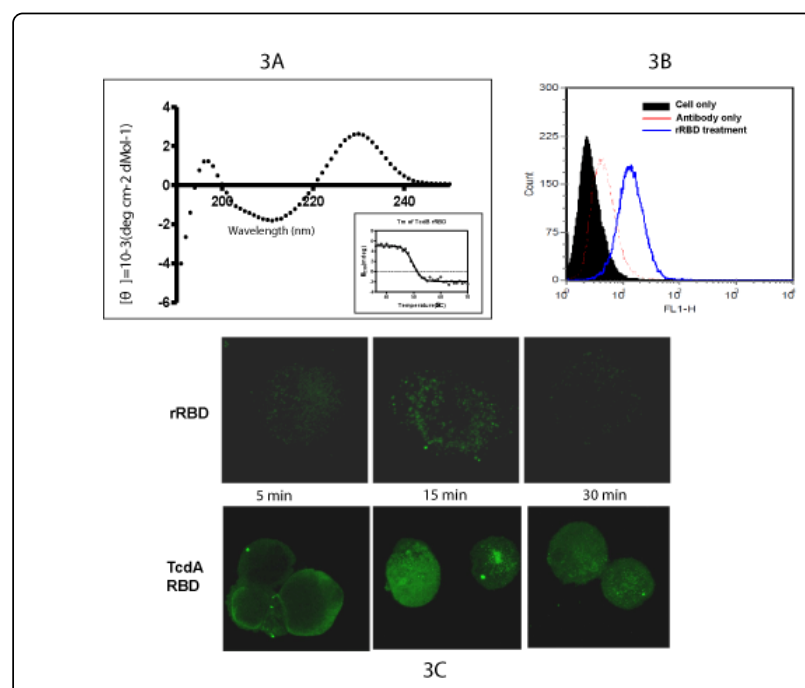

Figure 3: (A) CD spectra of rRBD. (B) Vero cell binding ability of rRBD was characterized by flow-cytometry. (C) Immunofluoresence signals of rRBD and TcdA RBD binding to Vero cell were analyzed by confocal micrscopy after 5,15 and 30 minutes rRBD inoculation.

\section{rRBD binding to Vero cells}

To further confirm that purified rRBD was correctly refolded, a Vero cell-binding assay was performed using flow cytometry. The results shown in Figure 3B indicate that within 5 minutes $\mathrm{rRBD}$ at a concentration of $0.3 \mu \mathrm{M}(20 \mu \mathrm{g} / \mathrm{mL})$ unambiguously binds to the Vero cell surface. rRBD also strongly binds to Caco-2 cells in a dosedependent manner (data not shown). As seen in Figure 3C, binding of TcdA RBD to Vero cell surfaces promotes its cellular internalization through receptor-mediated/clathrin-dependent pathway endocytosis [31]. To verify whether or not rRBD exhibited this biological activity, a Vero cell binding assay was performed and results were analyzed by immuno-fluorescence and confocal microscopy [31]. After 5 minutes, rRBD binding was detected as speckled immunofluorescent spots on the surface of Vero cells. This was different from what was observed with TcdA RBD where immuno-fluorescence signals were uniformly distributed over the cell surface (Figure 3C). This difference in cell surface binding patterns suggests that TcdA RBD and TcdB RBD may have different receptor-binding specificities. After 15 minutes of rRBD incubation, the immuno-fluorescence signals did not decrease but rather increased over time (Figure $3 \mathrm{C}$ ). This suggests that rRBD most 
likely had been internalized from the cell surface to the cytosol as shown by confocal microscopy (Figure 3C, 15 min picture). The results support rapid cell-binding of rRBD as illustrated by strong antirRBD fluorescence signals on the cell surface within 5 minutes and entry into cytosol after $15 \mathrm{~min}$ (Figure 3C, $15 \mathrm{~min}$ picture). In addition, the majority of rRBD inside the cytosol was degraded within 30 minutes (Figure 3C, 30 min picture). These experiments indicate that rRBD specifically binds to cell surfaces, is internalized into the cytosol, and then degraded within a short period of time. In contrast, the anti-TcdA RBD fluorescence signals were decreased, but not disappeared in 30 minutes (Figure 3C). This suggests that $\mathrm{rRBD}$ and TcdA RBD may internalize into different compartments, so the rate of degradation is different.

The hemagglutinin activity of rRBD was evaluated using rabbit erythrocytes. It was observed that $\mathrm{rRBD}$ could not agglutinate rabbit erythrocytes at concentrations as high as 2 nmoles. TcdA RBD used as the positive control easily agglutinated rabbit erythrocyte at a concentration of 2 pmoles [31]. Our result is consistent with the previous report [28] that recombinant TcdB RBD does not have HA activity.

\section{TcdB RBD can activate mouse dendritic cell maturation}

A and TcdA RBD have been reported to have the ability to upregulate cell surface marker expression and cytokine secretion from dendritic cells $[31,38]$. To further investigate whether rRBD could play a role in regulating the immune system, rRBD was tested for its ability to promote the maturation of mouse Dendritic Cells (DC). Bone marrow-derived DCs (BMDCs) from C57BL/6 mice were treated with increasing amounts $(0.6$ to $60 \mu \mathrm{g})$ of rRBD. Cell surface biomarkers associated with DC maturation (CD40, CD80, CD86, and MHC-II) and the secretion of pro-inflammatory cytokines (IL-6, IL12, and TNF- $\alpha$ ) were examined using FACS and cytokine-specific ELISA, respectively. In order to preclude the interference of LPS contamination, rRBD samples used in the studies contained very low amounts of residual LPS (0.03 EU/ $\mu \mathrm{g}$ of protein). In addition, polymyxin B was added to DC samples to prevent activation by LPS through the Toll-like receptor 4 pathway. It was found that surface biomarkers of dendritic cell maturation were up-regulated and that the production of pro-inflammatory cytokines (IL-6, IL-12, and TNF- $\alpha$ ) was significantly increased in a dose-dependent manner, from 0.2 to 2 $\mu \mathrm{M}$ (data not shown). Subsequent analyses and repeats were performed using a concentration of $1 \mu \mathrm{M}$ of $\mathrm{rRBD}$ in the final assay solution. DC maturation biomarkers were up-regulated (Figure 4A). The results were not influenced by LPS contamination since there was no significant difference between polymyxin B-treated and nontreated samples. A significant increase in the production of proinflammatory cytokines (IL-6, IL12, and TNF- $\alpha$ ) was detected in rRBD-treated BMDCs culture supernatant (Figure 4B). Interestingly, polymyxin $\mathrm{B}$ reduced at least $50 \%$ of the production of the proinflammatory cytokines (Figure 4B). To further confirm that the production of the pro-inflammatory cytokines was the result of treatment with rRBD, both rRBD and LPS were boiled for 10 minutes to destroy their biological functions. The boiling treatment did not affect LPS-induced DC activation but fully abolished rRBD DCactivation ability (data not shown). Combined these data clearly demonstrate that DC activation is mediated by rRBD. These results confirm that rRBD has intrinsic adjuvant functions that would be useful for its formulation as a candidate vaccine against CDAD.

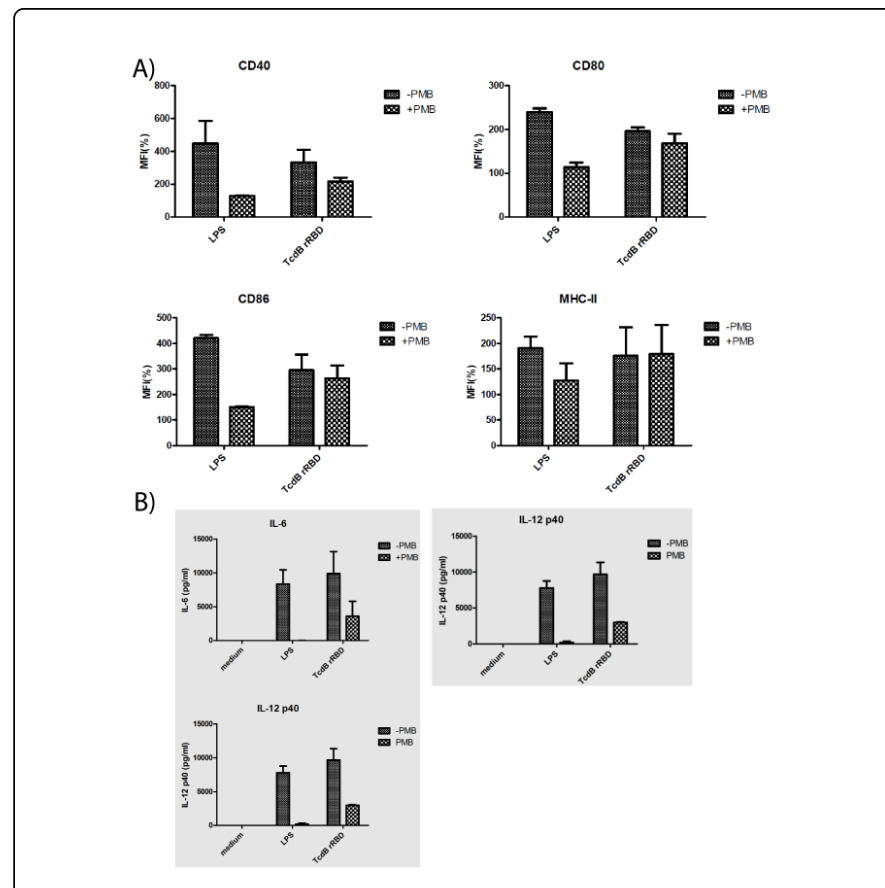

Figure 4: (A) Up-regulation of surface biomarkers on bone marrow-derived DC (BMDCs) by rRBD. BMDCs from C57BL/6 were collected and treated with GM-CSF at day 0 and 3. At day 6, rRBD ( $1 \mu \mathrm{M}$ final concentration) was added, and 18 hours later DCs were collected for surface biomarkers expression (including CD40, CD80, CD86, and MHC II) by flowcytometry. Samples were divided into groups treated or not treated with polymyxin $\mathrm{B}(\mathrm{PMB})$ to assess the influence of residual LPS contamination. Surface marker signaling was normalized by calculating the ratio of mean of fluorescence intensity (MFI) between medium control and treatments. (B) Cytokines secretion from BMDC treated with tcdA rRBD. After BMDCs were treated with rRBD at day 6 for 18 hours, culture supernatants were collected and analyzed for their cytokine profiles using specific cytokine ELISA: IL-6, IL12-p40, and TNF- $\alpha$.

\section{Immunogenicity of rRBD in mice}

To assess the immunogenicity of $\mathrm{rRBD}$, three groups of mice $(\mathrm{n}=6)$ were vaccinated with different amounts of $\mathrm{rRBD}$ alone $(0,3$ and 10 $\mu \mathrm{g})$. The results shown in Table 1 indicate that $\mathrm{rRBD}$ is a poor immunogen since mice vaccinated with $3 \times 3 \mu \mathrm{g}$ of $\mathrm{rRBD}$ alone produced poor anti-RBD IgG antibodies (titer $=800$ ) as measured by RBD-specific ELISA. Only antisera from mice immunized with 3 doses of $10 \mu \mathrm{g}$ rRBD alone induced significant antibody responses (titer $=3200 ; \mathrm{p}<0.05)$. Although antisera from mice vaccinated with $3 \times$ $10 \mu \mathrm{g}$ of $\mathrm{rRBD}$ showed both IgG1 and IgG2 isotypes antibody responses, systemic IgA antibody responses were not elicited by rRBD vaccination (data not shown).

To test whether mouse anti-RBD antibodies elicited by $\mathrm{rRBD}$ functionally neutralize the cytotoxicity of toxins (TcdA and TcdB), antisera were tested in a Vero cell cytotoxicity assay as described in Materials and Methods. As shown in Table 1, antisera from mice immunized with $3 \times 3 \mu \mathrm{g}$ of $\mathrm{rRBD}$ were found to have $1 / 16$ neutralization titer preventing $50 \%$ of cell death as a result of either toxin A or toxin B cytotoxicity. The neutralization titers obtained from 
Page 6 of 9

mice immunized with $3 \times 10 \mu \mathrm{g}$ of rRBD were found to be significantly high against toxin $B$ (titer $=64$; $\mathrm{p}<0.01$ ), but insignificant against toxin A as compared to those obtained from mice vaccinated with $3 \times 3 \mu \mathrm{g}$ dose (Tables 1). The current results indicate that $3 \times 10 \mu \mathrm{g}$ of $\mathrm{rRBD}$ alone was capable of inducing functional neutralizing antibodies against both toxins $\mathrm{A}$ (titer=16) and B (titer=64). Interestingly, the anti-RBD IgG antibody responses elicited by immunization with freeze-thaw or heat-treated $\mathrm{rRBD}$ were found to be significantly lower and have no neutralizing activity (data not shown). This shows the importance of preserving a functionally active conformation of rRBD.

\begin{tabular}{|c|c|c|c|c|}
\hline \multicolumn{2}{|c|}{$\begin{array}{l}\text { Mice immunized with various } \\
\text { amount of } \operatorname{rRBD}(\mu \mathrm{g})\end{array}$} & \multirow{3}{*}{$\begin{array}{l}\text { Anti-rRBD } \\
\text { titersa } \\
<100\end{array}$} & \multicolumn{2}{|c|}{$\begin{array}{l}\text { Neutralization } \\
\text { Titerb }\end{array}$} \\
\hline & & & \multirow{2}{*}{$\begin{array}{l}\text { TcdB } \\
<4\end{array}$} & \multirow{2}{*}{$\begin{array}{l}\text { TcdA } \\
<4\end{array}$} \\
\hline 0 & Pre-immune & & & \\
\hline & 2 doses & $<100$ & NP & NP \\
\hline & 3 doses & $<100$ & $<4$ & NP \\
\hline \multirow[t]{3}{*}{3} & Pre-immune & $<100$ & $<4$ & $<4$ \\
\hline & 2 doses & 400 & NP & NP \\
\hline & 3 doses & 800 & 16 & 16 \\
\hline \multirow[t]{3}{*}{10} & Pre-immune & $<100$ & $<4$ & $<4$ \\
\hline & 2 doses & 400 & NP & NP \\
\hline & 3 doses & 3200 & 64 & 16 \\
\hline TcdA RBDc & $3 \times 10 \mu g$ & 800 & $<4$ & 256 \\
\hline \multicolumn{5}{|c|}{ aThe titers were obtained with sera pooled from 6 mice } \\
\hline \multicolumn{5}{|c|}{$\begin{array}{l}\text { bThe neutralization titer against toxins was defined as the highest samp } \\
\text { dilution which could prevent } 50 \% \text { cell rounding induced by toxins }\end{array}$} \\
\hline \multicolumn{5}{|c|}{ cThe immunogenicity studies of TcdA RBD will be published elsewhere } \\
\hline \multicolumn{5}{|c|}{${ }^{*} \mathrm{NP}$ means not performed } \\
\hline
\end{tabular}

Table 1: $C$. difficile toxin B neutralization titers of antisera from groups of 6 mice immunized 3 times with varying amounts of rRBD.

\section{Hamster challenge studies}

To further evaluate the role of anti-toxin neutralizing antibodies in vivo, $C$. difficile spore challenge was performed in the hamster model as described in Materials and Methods. Three groups of hamsters $(n=6)$ were vaccinated 3 times, 2 weeks apart either with PBS, 3 or 10 $\mu \mathrm{g}$ of rRBD intramuscularlly. A week after the third immunization, blood samples collected from immunized hamsters were assayed for anti-TcdB neutralizing antibody titers and found to be $<4,16$ and 64 for PBS, 3 and $10 \mu \mathrm{g}$ dose groups, respectively. Interestingly, hamster antisera had no neutralizing activity against TcdA. Two weeks after the third immunizations hamsters were gastrically inoculated with 100 c.f.u. of $C$. difficile (lethal dose). Three to 4 days post challenge $100 \%$, $100 \%$ and $80 \%$ of hamsters died in the PBS, 3 and $10 \mu \mathrm{g}$ groups, respectively (Figure 5). This $20 \%$ partial protection was insignificant since in the repeated experiment, we observed $20 \%$ of survival rate in both PBS and $10 \mu \mathrm{g}$ of rRBD vaccinated hamster groups. In addition, significant amounts $\left(>1 \times 10^{3}\right.$ pfu per $\mu \mathrm{g}$ of faecal) of $C$. difficile colonized the selective TCCFA plates when faecal pellets collected from the surviving hamsters were analyzed. To test whether rRBD formulated with alum could elicit better protection in the hamster challenge model, group of hamsters were immunized $3 \times 10 \mu \mathrm{g}$ of rRBD formulated with alum and challenged gastrically with 100 c.f.u. of C. difficile. The anti-rRBD IgG antibodies were found to increase 10 to 50 folds (Titer $>5 \times 10^{4}$ ) but the neutralization titer remained to be 64 and protected 1 out of 6 hamsters in the challenge studies. These results are very similar to previous reports that TcdB and TcdB RBD did not confer protection in the hamster challenge model [22,27,37].

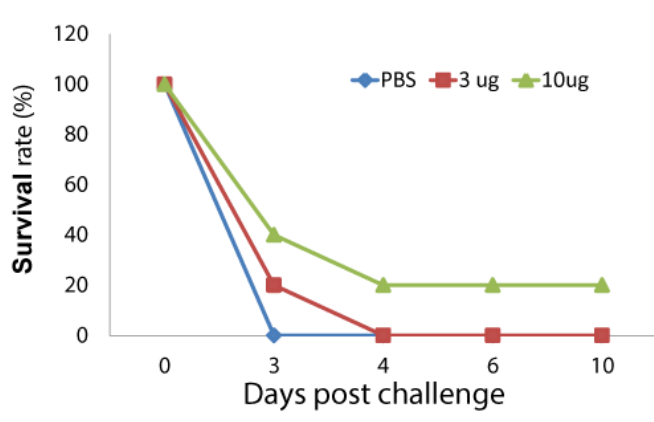

Figure 5: C. difficile spore challenge in hamster model studies. Four groups of hamsters $(n=6)$ were gastrically inoculated with 100 c.f.u. of $C$. difficile (lethal dose) at 2 weeks after the third immunizations of $\operatorname{rRBD}(0,3$, and $10 \mu \mathrm{g})$. The challenge studies are described in the Materials and Methods. Survival rates are recorded.

\section{Discussion}

Vaccine development against CDAD is urgently needed to control the rise of hospital-acquired $\mathrm{Cd}$ infections (CDI) which leads to excessive medical cost. TcdA toxoid-based vaccines against CDI are currently in phase III clinical trials $[9,18]$. The structural conformation of RBD undoubtedly correlates with its ability to elicit anti-toxin cross-neutralizing antibody responses. TcdA RBD is predicted to have $>900$ amino acids and 7 putative oligosaccharide (Gala1-3Gal $\beta 1-4$ GlcNAc) binding regions, whereas TcdB RBD has around 530 amino acids and 4 putative oligosaccharide binding sites [9,28-31,36]. Interestingly, among $C$. difficile strains deposited in the NCBI database, the amino acid sequences of the putative oligosaccharide binding sites of TcdA and TcdB shared about $50 \%$ identity or $70 \%$ similarity (Figure 6). To this end, we rationally designed two novel immunogens based on highly conserved protein sequences (90-97\%) of TcdA RBD and TcdB RBD which would potentially be capable of inducing broadly neutralizing functional antibodies against both toxins. The biochemical and immunological functions of TcdA RBD have been characterized and submitted for publication [31]. In the present study, we have successfully engineered and expressed a codon usage optimized tcdB RBD synthetic gene in $\mathrm{E}$. coli. More than $40 \mathrm{mg}$ of highly purified $\mathrm{rRBD}$ ( $>95 \%$ purity) were consistently obtained from 1 liter of bacterial culture after purification using Ni-affinity chromatography. Downstream purification including E-membrane filtration essentially eliminated LPS contamination $(<0.03 \mathrm{EU}$ of LPS per $\mu \mathrm{g}$ of protein). This level of LPS is known not to influence animal immunogenicity studies.

Several functional assays were performed to assess rRBD biological activities. Crystal structure studies revealed that disorder in the Cterminal binding domain of TcdA affects its biological function [29]. As a result, the purified $\mathrm{rRBD}$ was first analyzed and found to consist of a stable $\beta$-solenoid secondary structure as shown by $\mathrm{CD}$ spectra 
analysis (Figure 3A). This is consistent with previous reports $[28-29,31]$ that the majority of the secondary structure of rRBD is formed by $\beta$-solenoids (>40\%). The results obtained from Vero cellbinding assay as shown by FACS analysis and immuno-fluorescence assay (Figures $3 \mathrm{~B}$ and $3 \mathrm{C}$ ) provided direct evidence that $\mathrm{rRBD}$ is correctly folded to mediate its biological functions. Current immunofluorescence assays revealed that direct binding of rRBD to Vero cell surfaces occurs rapidly within 5 minutes. No loss of RBD-associated fluorescence signals in confocal microscopy indicated that RBD was transferred from the cell surface to the cytosol. The current result is consistent with a previous report [31] that TcdA RBD and its truncated fragments bind to Vero cell surfaces and enter the cytosol within 15 minutes of incubation. A point of interest is that TcdB RBD binds to specific receptor(s) on the surface of Vero cells as evidenced by the speckled pattern of the immuno-fluorescence signals. In contrast, TcdA RBD uniformly binds and covers the cell surface (Figure 3C, $5 \mathrm{~min}$ pictures). These different cell surface binding patterns indicate that TcdA RBD and TcdB RBD likely have different receptor-binding specificities as reported in previous studies [28-30,40]. In addition, a recent report [39] suggested that the Cterminal receptor binding domain of clostridial toxins mediates the first step of RBD-dependent endocytosis and that the translocation domain is required cell entry. To clarify the functions of translocation domain and putative carbohydrate-binding domains, the identification of natural cell-surface receptors are urgently needed

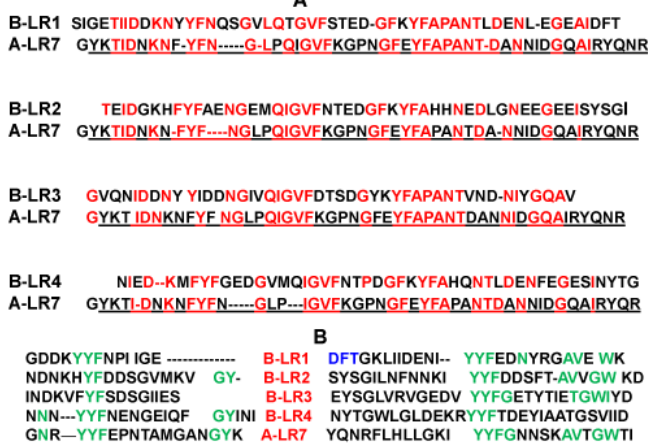

Figure 6: (A) Analysis of the putative oligosaccharide binding sites. A-LR7 (residues 795-844) and B-LR are the putative oligosaccharide binding sites of TcdA and TcdB, respectively. Since the crystal structure of A-LR7 was previously published [29], it was used for comparison with the putative oligosaccharide binding sites of TcdB. Identical amino acids are highlighted in red. (B) Amino acid sequence analysis of the regions in between the putative oligosaccharide binding sites of TcdB and LR7 of TcdA. Identical amino acids are highlighted in green.

Another point of interest from the current study is that rRBD shows comparable binding affinity for Vero cells $(100 \mathrm{nM})$ to TcdA RBD (50 $\mathrm{nM}$ ), but it does not agglutinate rabbit erythrocyte even at a 2 nmolar concentration, whereas TcdA RBD could easily agglutinate rabbit erythrocytes at 2 pmoles [31]. Since amino acid sequences of the putative oligosaccharide binding sites of TcdA and TcdB share no more than $70 \%$ similarity (Figure 6), differences in the cell-surface receptor-binding specificities of $\mathrm{Tcd} A$ and $\mathrm{TcdB}$ reported in the present and previous studies [28-30,40] may account for the lack of HA activity. Differences in amino acid sequences between the long- repeats are clearly seen when $\mathrm{TcdA}$ and $\mathrm{TcdB}$ sequences are compared (Figure 6B). It will be of interest to study the specific role(s) and function(s) of each putative oligosaccharide binding region and to test whether $\mathrm{rRBD}$ and/or its fragments have the same cell-surface receptor-binding specificities. There is a need to identify the natural cell-surface receptors.

Both TcdA and cholera toxin had been shown to stimulate endothelial cells and DC activation [38,41,43], such activation has been confirmed to be the result of TcdA RBD [31,41]. In our current study, we have clearly shown that $1 \mu \mathrm{M}$ of $\mathrm{rRBD}$ promoted the maturation of BMDC and an increased secretion of pro-inflammatory cytokines (Figure 4). rRBD functions as a toll-like receptor (TLR) agonist that was capable of enhancing immune responses. This was supported by the fact that rRBD in the absence of adjuvant elicits neutralizing antibody responses. It will be of interest to determine whether $\mathrm{rRBD}$ at $10 \mu \mathrm{g}$ doses can effectively enhance specific IgG antibody responses against poor immunogens as there is strong evidence that rRBD has intrinsic immuno-stimulatory properties.

To obtain maximum protective efficacy, TcdA RBD and/or its fragments were formulated with different types of adjuvant such as alum, MF59, CFA/IFA, flagellin, heat-labile enterotoxin from Eschelichia coli (LT) and cholera toxin from Vibrio cholerae (CT), and/or vaccine delivery systems including recombinant vectors based on adenovirus, Vibrio cholerae and bacillus spores [21-26,44-47]. In these studies, high dosages of recombinant TcdA RBD and/or RBD fragments $(100 \mu \mathrm{g})$ were intraperitoneally injected into animals to generate neutralizing antibodies against CDI. In contrast to these previous studies, results in Table 1 have shown that $10 \mu \mathrm{g}$ of rRBD alone elicited antibody responses that inhibited the cytotoxicity of $\mathrm{Tcd} A$ and $\mathrm{TcdB}$ in vitro. This result unambiguously demonstrates that rRBD can be a component of potential vaccine candidates against CDAD.

\begin{tabular}{|c|c|c|c|c|c|c|c|}
\hline \multirow{3}{*}{ Antigen } & \multicolumn{3}{|c|}{ Biological Properties $^{a}$} & \multicolumn{4}{|c|}{ Immunological Properties ${ }^{b}$} \\
\hline & \multirow[b]{2}{*}{$\begin{array}{l}\text { Cell- } \\
\text { binding }\end{array}$} & \multirow[b]{2}{*}{$\begin{array}{l}\text { HA } \\
\text { activity }\end{array}$} & \multirow[b]{2}{*}{$\begin{array}{l}\text { Cell } \\
\text { entry }\end{array}$} & \multicolumn{2}{|c|}{$\begin{array}{l}\text { Elicit } \\
\text { neutralizing } \\
\text { antibodies }\end{array}$} & \multirow{2}{*}{$\begin{array}{l}\text { Elicit } \\
\text { protection } \\
\text { in } \\
\text { hamster } \\
\text { model }\end{array}$} & \multirow[t]{2}{*}{$\begin{array}{l}\text { Activate } \\
\text { dendritic } \\
\text { cell }\end{array}$} \\
\hline & & & & TcdA & TcdB & & \\
\hline $\begin{array}{l}\text { TcdA } \\
\text { RBD }\end{array}$ & $50 \mathrm{nM}$ & $\begin{array}{l}0.4 \\
\text { pmoles }\end{array}$ & Fast & 256 & $<4$ & No & $0.4 \mu \mathrm{M}$ \\
\hline $\begin{array}{l}\text { TcdB } \\
\text { RBD }\end{array}$ & $100 \mathrm{nM}$ & No & Fast & 16 & 64 & No & $1 \mu \mathrm{M}$ \\
\hline TcdB & Yes $^{* *}$ & NP & Yes & NP & Yes & $\mathrm{NP}$ & NP \\
\hline
\end{tabular}

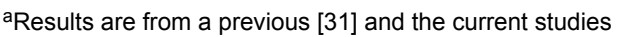

bThe immunogenicity studies of TcdA RBD will be published elsewhere

*NP means not performed

${ }^{* *}$ Yes refers to information from the literature [7-23,31].

Table 2: Summary of biochemical and immunological properties of TcdA RBD and TcdB RBD.

In summary, the recombinant TcdB RBD based on $C$. difficile strain VPI10463 with $>95 \%$ amino acid sequence identity to hyper-virulent 
strain BI/NAP1/027 was successfully expressed in Escherichia coli, purified, characterized biologically and immunologically and found to have the following properties (Table 2): (a) capable of binding to the cell surface of both Vero and Caco-2 cells and entry into the cytosol; (b) devoid of hemagglutinin activity (HA); (c) functioning as a toll-like receptor agonist activating dendritic cell maturation; and (d) in the absence of adjuvant eliciting anti-TcdB neutralizing antibody responses that weakly cross-neutralize TcdA. As rRBD could induced weak protection in hamster challenge studies, rRBD will be formulated with TcdA RBD and/or other domains of TcdB in the present of adjuvant (alum) and tested whether it is potentially a good immunogen candidate to be included in future vaccines against Clostridium difficile-associated diseases.

\section{Acknowledgements}

This work was supported by grants from the Ministry of Science and Technology of Taiwan (NSC 101-2320-B-400-012 and NSC 101-2923-B-400-001-MY3) and the National Health Research Institutes, Taiwan. The authors thank Professor Michel Klein and Miss Lori Chong for their comments on the manuscript.

\section{References}

1. Knoop FC, Owens M, Crocker IC (1993) Clostridium difficile: clinical disease and diagnosis. Clin Microbiol Rev 6: 251-265.

2. Lyerly DM, Krivan HC, Wilkins TD (1988) Clostridium difficile: its disease and toxins. Clin Microbiol Rev 1: 1-18.

3. Kelly CP, LaMont JT (2008) Clostridium difficile--more difficult than ever. N Engl J Med 359: 1932-1940.

4. McDonald LC, Killgore GE, Thompson A, Owens RC Jr, Kazakova SV, et al. (2005) An epidemic, toxin gene-variant strain of Clostridium difficile. N Engl J Med 353: 2433-2441.

5. He M, Miyajima F, Roberts P, Ellison L, Pickard DJ, et al. (2013) Emergence and global spread of epidemic healthcare-associated Clostridium difficile. Nat Genet 45: 109-113.

6. Sun X, Savidge T, Feng H (2010) The enterotoxicity of Clostridium difficile toxins. Toxins (Basel) 2: 1848-1880.

7. Kuehne SA, Cartman ST, Heap JT, Kelly ML, Cockayne A, et al. (2010) The role of toxin A and toxin B in Clostridium difficile infection. Nature 467: 711-713.

8. Davies AH, Roberts AK, Shone CC, Acharya KR (2011) Super toxins from a super bug: structure and function of Clostridium difficile toxins. Biochem J 436: 517-526.

9. Leuzzi R, Adamo R, Scarselli M (2014) Vaccines against Clostridium difficile. Hum Vaccin Immunother 10: 1466-1477.

10. Kim PH, Iaconis JP, Rolfe RD (1987) Immunization of adult hamsters against Clostridium difficile-associated ileocecitis and transfer of protection to infant hamsters. Infect Immun 55: 2984-2992.

11. Kyne L, Warny M, Qamar A, Kelly CP (2001) Association between antibody response to toxin $\mathrm{A}$ and protection against recurrent Clostridium difficile diarrhoea. Lancet 357: 189-193.

12. Leav BA, Blair B, Leney M, Knauber M, Reilly C, et al. (2010) Serum antitoxin B antibody correlates with protection from recurrent Clostridium difficile infection (CDI). Vaccine 28: 965-969.

13. Davies NL, Compson JE, Mackenzie B, O'dowd VL, Oxbrow AK, et al. (2013) A mixture of functionally oligoclonal humanized monoclonal antibodies that neutralize Clostridium difficile TcdA and TcdB with high levels of in vitro potency shows in vivo protection in a hamster infection model. Clin Vaccine Immunol 20: 377-390.

14. Marozsan AJ, Ma D, Nagashima KA, Kennedy BJ, Kang YK, et al. (2012) Protection against Clostridium difficile infection with broadly neutralizing antitoxin monoclonal antibodies. J Infect Dis 206: 706-713.
15. Babcock GJ, Broering TJ, Hernandez HJ, Mandell RB, Donahue K, et al. (2006) Human monoclonal antibodies directed against toxins A and B prevent Clostridium difficile-induced mortality in hamster. Infect Immun 74: 6339-6347.

16. Kink JA, Williams JA (1998) Antibodies to recombinant Clostridium difficile toxins $\mathrm{A}$ and $\mathrm{B}$ are an effective treatment and prevent relapse of C. difficile-associated disease in a hamster model of infection. Infect Immun 66: 2018-2025.

17. Wang H, Sun X, Zhang Y, Li S, Chen K, et al. (2012) A chimeric toxin vaccine protects against primary and recurrent Clostridium difficile infection. Infect Immun 80: 2678-2688

18. Anosova NG, Brown AM, Li L, Liu N, Cole LE, et al. (2013) Systemic antibody responses induced by a two-component Clostridium difficile toxoid vaccine protect against C. difficile-associated disease in hamsters. J med microbiol 62: 1394-1404.

19. Torres JF, Lyerly DM, Hill JE, Monath TP. (1995) Evaluation of formalin-inactivated Clostridium difficile vaccines administered by parenteral and mucosal routes of immunization in hamsters. Infect Immun 63: 4619-4627.

20. Sauerborn M, Leukel P, von Eichel-Streiber C (1997) The C-terminal ligand-binding domain of Clostridium difficile toxin A (TcdA) abrogates TcdA-specific binding to cells and prevents mouse lethality. FEMS Microbiol Lett 155: 45-54.

21. Seregin SS, Aldhamen YA, Rastall DP, Godbehere S, Amalfitano A (2012) Adenovirus-based vaccination against Clostridium difficile toxin A allows for rapid humoral immunity and complete protection from toxin A lethal challenge in mice. Vaccine 30: 1492-1501.

22. Tian JH, Fuhrmann SR, Kluepfel-Stahl S, Carman RJ, Ellingsworth L, et al. (2012) A novel fusion protein containing the receptor binding domains of C. difficile toxin A and toxin B elicits protective immunity against lethal toxin and spore challenge in preclinical efficacy models. Vaccine 30: 4249-4258.

23. Ryan ET, Butterton JR, Smith RN, Carroll PA, Crean TI, et al. (1997) Protective immunity against Clostridium difficile toxin A induced by oral immunization with a live, attenuated Vibrio cholerae vector strain. Infect Immun 65: 2941-2949.

24. Ward SJ, Douce G, Figueiredo D, Dougan G, Wren BW (1999) Immunogenicity of a Salmonella typhimurium aroA aroD vaccine expressing a nontoxic domain of Clostridium difficile toxin A. Infect Immun 67: 2145-2152.

25. Gardiner DF, Rosenberg T, Zaharatos J, Franco D, Ho DD (2009) A DNA vaccine targeting the receptor-binding domain of Clostridium difficile toxin A. Vaccine 27: 3598-3604.

26. Permpoonpattana P, Hong HA, Phetcharaburanin J, Huang JM, Cook J, et al. (2011) Immunization with Bacillus spores expressing toxin A peptide repeats protects against infection with Clostridium difficile strains producing toxins A and B. Infect Immun 79: 2295-2302.

27. Leuzzi R, Spencer J, Buckley A, Brettoni C, Martinelli M, et al. (2013) Protective efficacy induced by recombinant Clostridium difficile toxin fragments. Infect Immun 81: 2851-2860.

28. Dingle T, Wee S, Mulvey GL, Greco A, Kitova EN, et al. (2008) Functional properties of the carboxy-terminal host cell-binding domains of the two toxins, TcdA and TcdB, expressed by Clostridium difficile. Glycobiology 18: 698-706.

29. Ho JG, Greco A, Rupnik M, Ng KK (2005) Crystal structure of receptorbinding C-terminal repeats from Clostridium difficile toxin A. Proc Natl Acad Sci U S A 102: 18373-18378.

30. Krivan HC, Clark GF, Smith DF, Wilkins TD (1986) Cell surface binding site for Clostridium difficile enterotoxin: evidence for a glycoconjugate containing the sequence Gal alpha 1-3Gal beta 1-4GlcNAc. Infect Immun 53: 573-581.

31. Huang JH, Shen ZQ, Lien SP, Hsiao KN, Leng CH, et al. (2014) Biochemical characterizations of the receptor binding domains of $\mathrm{C}$. difficile Toxin A.

32. Greenfield N, Fasman GD (1969) Computed circular dichroism spectra for the evaluation of protein conformation. Biochemistry 8: 4108-4116. 
Citation: Huang J, Wu C, Lien S, Hsiao K, Leng C, et al. (2015) Biochemical and Immunological Characterizations of the Receptor Binding Domain of C. difficile Toxin B. J Vaccines Vaccin 6: 276. doi:10.4172/2157-7560.1000276

Page 9 of 9

33. Wren BW, Russell RR, Tabaqchali S (1991) Antigenic cross-reactivity and functional inhibition by antibodies to Clostridium difficile toxin A Streptococcus mutans glucan-binding protein, and a synthetic peptide. Infect Immun 59: 3151-3155.

34. Lutz MB, Kukutsch N, Ogilvie AL, Rössner S, Koch F, et al. (1999) An advanced culture method for generating large quantities of highly pure dendritic cells from mouse bone marrow. J Immunol Methods 223: 77-92.

35. Lyras D, O'Connor JR, Howarth PM, Sambol SP, Carter GP, et al. (2009) Toxin $\mathrm{B}$ is essential for virulence of Clostridium difficile. Nature 458 1176-1179.

36. Stabler RA, Dawson LF, Phua LT, Wren BW (2008) Comparative analysis of BI/NAP1/027 hypervirulent strains reveals novel toxin B-encoding gene (tcdB) sequences. J Med Microbiol 57: 771-775.

37. Papatheodorou P, Zamboglou C, Genisyuerek S, Guttenberg G, Aktories $\mathrm{K}$ (2010) Clostridial glucosylating toxins enter cells via clathrin-mediated endocytosis. PLoS One 5: e10673.

38. Lee JY, Kim H, Cha MY, Park HG, Kim YJ, et al. (2009) Clostridium difficile toxin A promotes dendritic cell maturation and chemokine CXCL2 expression through p38, IKK, and the NF-kappaB signaling pathway. J Mol Med (Berl) 87: 169-180.

39. Gerhard R, Frenzel E, Goy S, Olling A (2013) Cellular uptake of Clostridium difficile TcdA and truncated TcdA lacking the receptor binding domain. J Med Microbiol 62: 1414-1422.

40. Chaves-Olarte E, Weidmann M, Eichel-Streiber C, Thelestam M (1997) Toxins $\mathrm{A}$ and $\mathrm{B}$ from Clostridium difficile differ with respect to enzymatic potencies, cellular substrate specificities, and surface binding to cultured cells. J Clin Invest 100: 1734-1741.
41. Yeh CY, Lin CN, Chang CF, Lin CH, Lien HT, et al. (2008) C-terminal repeats of Clostridium difficile toxin $\mathrm{A}$ induce production of chemokine and adhesion molecules in endothelial cells and promote migration of leukocytes. Infect Immun 76: 1170-1178.

42. Gagliardi MC, Sallusto F, Marinaro M, Langenkamp A, Lanzavecchia A, et al. (2000) Cholera toxin induces maturation of human dendritic cells and licences them for Th2 priming. Eur J Immunol 30: 2394-2403.

43. Lavelle EC, Grant G, Pusztai A, Pfüller U, O'Hagan DT (2001) The identification of plant lectins with mucosal adjuvant activity. Immunology 102: 77-86.

44. Ward SJ, Douce G, Dougan G, Wren BW (1999) Local and systemic neutralizing antibody responses induced by intranasal immunization with the nontoxic binding domain of toxin A from Clostridium difficile. Infect Immun 67: 5124-5132.

45. Artiushin S, Timoney JF, Fettinger M, Fallon L, Rathgeber R (2013) Immunisation of mares with binding domains of toxins $\mathrm{A}$ and $\mathrm{B}$ of Clostridium difficile elicits serum and colostral antibodies that block toxin binding. Equine Vet J 45: 476-480.

46. Yoshino Y, Kitazawa T, Ikeda M, Tatsuno K, Yanagimoto S, et al. (2013) Clostridium difficile flagellin stimulates toll-like receptor 5, and toxin $\mathrm{B}$ promotes flagellin-induced chemokine production via TLR5. Life Sci 92: 211-217.

47. Castagliuolo I, Sardina M, Brun P, DeRos C, Mastrotto C, et al. (2004) Clostridium difficile toxin A carboxyl-terminus peptide lacking ADPribosyltransferase activity acts as a mucosal adjuvant. Infect Immun 72: 2827-2836. 